\title{
An Unusual Case of Deep Vein Thrombosis and Mycotic Aneurysms Secondary to Salmonella Bacteraemia
}

\author{
Malarkodi Suppamutharwyam¹, Anuradha P. Radhakrishnan² \\ ${ }^{1}$ Department of Internal Medicine, Sarikei General Hospital, Sarikei, Malaysia \\ ${ }^{2}$ Department of Internal Medicine, Selayang General Hospital, Batu Caves, Malaysia \\ Email: malar2407@gmail.com, anurapr70@gmail.com
}

How to cite this paper: Suppamutharwyam, M. and Radhakrishnan, A.P. (2022) An Unusual Case of Deep Vein Thrombosis and Mycotic Aneurysms Secondary to Salmonella Bacteraemia. Case Reports in Clinical Medicine, 11, 37-47.

https://doi.org/10.4236/crcm.2022.113007

Received: January 29, 2022

Accepted: March 1, 2022

Published: March 4, 2022

Copyright $\odot 2022$ by author(s) and Scientific Research Publishing Inc. This work is licensed under the Creative Commons Attribution International License (CC BY 4.0).

http://creativecommons.org/licenses/by/4.0/

\section{(c) (i) Open Access}

\begin{abstract}
Salmonella, a food-borne pathogen, can cause mild self-limiting gastroenteritis. However, immunocompromised hosts and older adults with complex medical conditions may develop a complicated form of bacteraemia, with a high mortality rate involving extra-intestinal foci of infection and mycotic aneurysms. We report the case of a 61-year-old man with poorly controlled diabetes mellitus, hypertension, dyslipidaemia, and congestive heart failure, who presented with unilateral left lower limb swelling, extensive deep vein thrombosis, and concomitant Salmonella bacteraemia. An oral anticoagulant and intravenous antibiotic therapy were initiated. Although the patient remained haemodynamically stable, he complained of constant left lower limb weakness and lower back pain. A computed tomography angiography scan of the thorax and abdomen revealed saccular aneurysms with contained hematoma of the left common iliac artery. The oral anticoagulant was discontinued, and an inferior vena cava filter was inserted as part of the venous thrombosis management. The patient was offered aorto-uni-iliac endovascular aneurysm repair and received intravenous antibiotic therapy, postoperatively, for six weeks. The postoperative blood cultures remained negative, and he was discharged with a course of ciprofloxacin administered orally. However, three months after the surgery, the patient died of recurrent septicaemia. This case illustrates the importance of remaining vigilant for potential endovascular complications of Salmonella bacteraemia, such as mycotic aneurysms and deep vein thrombosis, among high-risk patients. Further, this case highlights the challenges of eliminating Salmonella bacteraemia and its related complications, albeit treating it with both a prolonged course of medical therapy and surgical intervention.
\end{abstract}




\section{Keywords}

Deep Vein Thrombosis, Endovascular Aneurysm Repair, Infective

Endarteritis, Mycotic Aneurysm, Salmonella

\section{Introduction}

Gastroenteritis is a common clinical feature of Salmonella infections and is often self-limiting, without complications. However, approximately $5 \%$ of affected patients develop Salmonella bacteraemia [1]. Disseminated infections are more likely to occur in immunocompromised hosts and older adults with complex medical conditions [2] [3] [4] [5]. These patients may develop complications involving endovascular infection and deep bone and visceral abscesses, which are challenging to treat and have a high mortality rate [6]. A recent study reported that Salmonella bacteraemia is the second most common cause of mycotic aneurysms and that its incidence is higher in Asian countries than in the West [7] [8]. In addition, Salmonella bacteraemia can present with atypical features, such as deep vein thrombosis [9] [10] [11]. Awareness of the rare and unusual clinical presentations of invasive Salmonella infection is essential for accurate diagnosis and prompt management.

Therefore, clinicians caring for high-risk patients with complex medical conditions need to remain vigilant and perform comprehensive evaluations to assess potential complications of Salmonella bacteraemia, such as mycotic aneurysms and deep vein thrombosis. Mycotic aneurysms involving multiple anatomical sites are challenging to treat, even when using a combination of medical and surgical interventions. Negative blood cultures, after a specific duration of antibiotic therapy and in the postoperative period, do not necessarily indicate complete bacterial elimination.

Here, we present the case of a 61-year-old man who presented with extensive deep vein thrombosis of the left lower limb with concomitant Salmonella bacteraemia and subsequently developed mycotic aneurysms.

\section{Case Presentation}

A 61-year-old man, with poorly controlled diabetes mellitus (Haemoglobin A1c: $12.5 \%)$, hypertension, dyslipidaemia, and congestive heart failure, presented with a gradual onset of unilateral left lower limb swelling for two weeks, which rendered him unable to walk. He had not been previously investigated for ischaemic causes of heart failure and had defaulted follow-up. He reported a subjective feeling of shortness of breath and had symptoms equivalent to the New York Heart Association Class III that were limiting his daily activities. He also reported significant tobacco use (smoking history of 40 pack-years).

On admission, his vitals were stable, with a low-grade fever of $37.6^{\circ} \mathrm{C}$; blood pressure of 128/78 $\mathrm{mmHg}$; pulse rate of 96 beats/min; respiratory rate of 18 
breaths/min and oxygen saturation of $100 \%$ breathing ambient air. Examination of the left lower limb revealed asymmetric calf swelling of more than $3 \mathrm{~cm}$ compared to the contralateral leg, without any pus discharge or skin excoriation. The active range of movement of the left hip, knee, and ankle was limited, owing to pain. Blood investigations revealed a white blood cell count of $9.46 \times 10^{3} / \mu \mathrm{L}$ with a neutrophil predominance of $77 \%$ and an erythrocyte sedimentation rate of 79 $\mathrm{mm} / \mathrm{h}$ (Table 1). Based on the elevated inflammatory markers and low-grade fever, a presumptive diagnosis of left lower limb cellulitis was made, and the patient was started on intravenous ceftriaxone. A chest radiography scan revealed cardiomegaly. Based on elevated D-dimer levels of $2251 \mathrm{ng} / \mathrm{ml}$ and a Wells score of 3 , an urgent lower-extremity venous ultrasound examination revealed extensive thrombi in the left common femoral, superficial femoral, and popliteal veins (Table 1). There were no focal loculated fluid collections indicative of septic arthritis in the hip, knee, or ankle joints. He denied any long-distance travel, recent surgery, or a malignancy history. To prevent further clotting and an embolism, he was started on subcutaneous enoxaparin and bridged with dabigatran.

Table 1. Progress of patient's relevant blood investigations throughout hospital admission.

\begin{tabular}{|c|c|c|c|c|c|c|}
\hline & Normal Values & Day 1 & Day 7 & Day 14 & Day 28 & Day 42 \\
\hline $\operatorname{WCC}\left(10^{3} / \mathrm{uL}\right)$ & $3.60-10.20$ & 9.46 & 13.71 & 7.14 & 7.90 & 9.9 \\
\hline Neutrophil percentage & $43 \%-73 \%$ & $77 \%$ & $80.6 \%$ & $74 \%$ & $70 \%$ & $72 \%$ \\
\hline Haemoglobin (g/dL) & $12.5-16.0$ & 11.2 & 10.5 & 9.2 & 7.8 & 7.7 \\
\hline MCV (fL) & $80-100$ & 79.3 & 75 & 75 & 81.6 & 90 \\
\hline $\mathrm{MCH}(\mathrm{pg})$ & $27-33$ & 27 & 27 & 26 & 27 & 26 \\
\hline Platelet $\left(10^{3} / \mathrm{uL}\right)$ & $152-347$ & 188 & 335 & 292 & 311 & 279 \\
\hline $\operatorname{ESR}(\mathrm{mm} / \mathrm{h})$ & $\leq 15 \mathrm{~mm} / \mathrm{h}$ & 79 & 49 & 100 & 104 & 64 \\
\hline D-Dimer(ng/ml) & $0-253.5$ & 2251 & & & & \\
\hline $\mathrm{PT}(\mathrm{sec})$ & $11-16$ & 16.1 & 17 & 20 & 14.7 & 15.9 \\
\hline PTT (sec) & $30-40$ & 54.3 & 52 & 74 & 49.7 & 61.9 \\
\hline INR & & 1.3 & 1.3 & 1.4 & 1.2 & 1.3 \\
\hline Urea (mmol/L) & $1.7-8.3$ & 8.2 & 7.8 & 3.7 & 2.9 & 4.0 \\
\hline Creatinine (umol/L) & $80-115$ & 83 & 97 & 67 & 75 & 78 \\
\hline Sodium (mmol/L) & $133-145$ & 126 & 131 & 128 & 128 & 134 \\
\hline Potassium (mmol/L) & $3.3-5.1$ & 4.7 & 4.1 & 3.8 & 4.3 & 3.7 \\
\hline Albumin $(\mathrm{g} / \mathrm{L})$ & $38-51$ & 24 & 24 & 21 & 29 & 29 \\
\hline AST (U/L) & $5-41$ & 116 & 93 & 73 & 60 & 63 \\
\hline $\operatorname{ALT}(\mathrm{U} / \mathrm{L})$ & $5-37$ & 93 & 45 & 31 & 30 & 25 \\
\hline Hepatitis B \& C & & NR & & & & \\
\hline HIV & & NR & & & & \\
\hline Syphilis & & NR & & & & \\
\hline
\end{tabular}


Continued

\begin{tabular}{|c|c|c|c|c|}
\hline Stool Culture & & $\mathrm{Neg}$ & Neg & \\
\hline Blood Culture and Susceptibility & & $\begin{array}{l}\text { Salmonella enteritidis } \\
\text { Susceptible to: } \\
\text { - Ceftriaxone } \\
\text { - Ampicillin } \\
\text { - Co-trimoxazole }\end{array}$ & Neg & Neg \\
\hline PSA (ng/mL) & $1-1.5$ & 0.9 & & \\
\hline CEA (ng/mL) & $0-2.5$ & 0.21 & & \\
\hline $\operatorname{AFP}(\mathrm{ng} / \mathrm{mL})$ & $10-20$ & 4 & & \\
\hline CA $19-9(\mathrm{U} / \mathrm{mL})$ & $0-37$ & 2.1 & & \\
\hline ANA & & Neg & & \\
\hline Anti-ds DNA & & Neg & & \\
\hline RF & & Neg & & \\
\hline Direct \& Indirect Coomb's & & Neg & & \\
\hline Haemoglobin A1C & $<6.0 \%$ & $12.5 \%$ & & \\
\hline Total Cholesterol (mmol/L) & $0.1-5.2$ & 5.4 & & \\
\hline $\mathrm{HDL}(\mathrm{mmol} / \mathrm{L})$ & $0.91-3.12$ & 1.25 & & \\
\hline $\mathrm{LDL}(\mathrm{mmol} / \mathrm{L})$ & $0.9-3.9$ & 4.2 & & \\
\hline Triglycerides (mmol/L) & $0.1-2.27$ & 1.1 & & \\
\hline
\end{tabular}

Abbreviations: AFP, alpha-fetoprotein; ALT, alanine aminotransferase; ANA, anti-nuclear antibodies; Anti dsDNA, antidouble-stranded DNA antibody; AST, aspartate aminotransferase; CA 19-9, cancer antigen 19-9; CEA, carcinoembryonic antigen; ESR, erythrocyte sedimentation rate; HIV, human immunodeficiency virus; INR, international normalized ratio; $\mathrm{MCH}$, mean corpuscular haemoglobin; MCV, mean corpuscular volume; Neg, negative; NR, non-reactive; PTT, partial thromboplastin time; PT, prothrombin time; PSA, prostate specific antigen; RF, rheumatoid factor; WCC, white cell count.

A preliminary report showed a gram-negative organism in the blood culture. Intravenous ceftriaxone was continued, and the patient remained haemodynamically stable and afebrile. The inflammatory markers improved, whereby the erythrocyte sedimentation rate decreased from $79 \mathrm{~mm} / \mathrm{h}$ to $49 \mathrm{~mm} / \mathrm{h}$ (Table 1 ). However, the patient continued to report a subjective feeling of fatigue and constant lower back pain, with a pain score of $4 / 10$, which did not improve even after receiving intravenous antibiotics for a few days. Later, it became apparent that he was harbouring Salmonella enteritidis, which was susceptible to ampicillin, ceftriaxone, and co-trimoxazole (Table 1). The patient was co-managed by an infectious disease team, and the antibiotic was changed to intravenous ampicillin.

Collaborative history from the patient and his family members did not reveal prior gastroenteritis symptoms and significant risk factors for infection, such as travel, consumption of fast foods, poultry, raw eggs, or contact with pets were absent. The patient denied having constitutional symptoms, weight loss, or a prior history of thrombosis, and his family history was unremarkable. The stool culture and susceptibility tests were negative (Table 1 ). Infective screening for 
hepatitis B and C, human immunodeficiency virus, and syphilis were negative (Table 1). Extensive investigations, including tumour markers and computed tomography (CT) scans of the thorax and abdomen, did not reveal any evidence of a solid organ tumour (Table 1). A peripheral blood smear revealed microcytic hypochromic anaemia but otherwise unremarkable for haematological malignancies. The result of comprehensive panel of rheumatological markers also tested negative (Table 1).

During the second week post admission, the patient reported worsening of lower back pain that hindered his walking abilities. The neurological examination was unremarkable, with localised pain over the left gluteal area and a severely restricted range of movement of the left hip owing to pain. The patient continued to remain haemodynamically stable and afebrile. Blood investigations revealed worsening inflammatory markers with a white blood cell count of 7.14 $\times 10^{3} / \mu \mathrm{L}$ with a neutrophil predominance of $74 \%$ and an elevated erythrocyte sedimentation rate of $100 \mathrm{~mm} / \mathrm{h}$. The patient's haemoglobin levels decreased from $11.2 \mathrm{~g} / \mathrm{dL}$ to $9.2 \mathrm{~g} / \mathrm{dL}$. An urgent esophago-gastro-duodenoscopy and colonoscopy did not reveal active gastrointestinal bleeding. Subsequently, an urgent multi-slice spiral CT combined with computed tomography angiography (CTA) of the thorax, abdomen, and pelvis was performed to identify potential invasive complications of Salmonella bacteraemia such as discitis, psoas abscess, and mycotic aneurysms. The CTA scan of the thorax, abdomen and pelvis revealed the presence of a $1.0 \mathrm{~cm}$ anteroposterior $(\mathrm{AP}) \times 0.8 \mathrm{~cm}$ width $(\mathrm{WT})$ saccular aneurysm at superolateral wall and $5.7 \mathrm{~cm}(\mathrm{AP}) \times 7.7 \mathrm{~cm}(\mathrm{WT}) \times 6.4 \mathrm{~cm}$ craniocaudal (CC) lobulated saccular aneurysm at the inferomedial wall of the left common iliac artery with contained hematoma (Figure 1 and Figure 2). Large saccular aneurysm does laterally displace the psoas muscle and compress the left distal ureter, which results in mild proximal hydroureter and hydronephrosis. The CTA abdomen also revealed infrarenal dissecting abdominal aortic aneurysm (AAA) measures $3.2 \mathrm{~cm}$ in its widest diameter (Figure 1). The true and false lumens measure $1.6 \mathrm{~cm}$ in diameter respectively. An echocardiogram revealed an ejection fraction of $20 \%$, with hypokinesia in the apical and septal regions, with no evidence of valvular vegetation.

With these observations, the oral anticoagulant was discontinued due to the risk of aneurysmal rupture. As an alternative, the patient received an inferior vena cava filter to prevent embolic events from the extensive left lower-limb deep vein thrombosis. Further discussions occurred with the vascular team about the surgical interventions. After six weeks of intravenous antibiotic therapy and negative blood cultures, the patient was transferred to their facility. Considering the patient's poor cardiac reserve and multiple comorbidities, which carry an enhanced perioperative risk, the patient was offered aorto-uni-iliac endovascular aneurysm repair (EVAR) of the left common iliac artery. Postoperatively, the patient remained haemodynamically stable, received six weeks of intravenous ceftazidime, and had negative blood cultures with no evidence of leakage. However, endovascular cultures were not evaluated. At six-weeks post EVAR and 


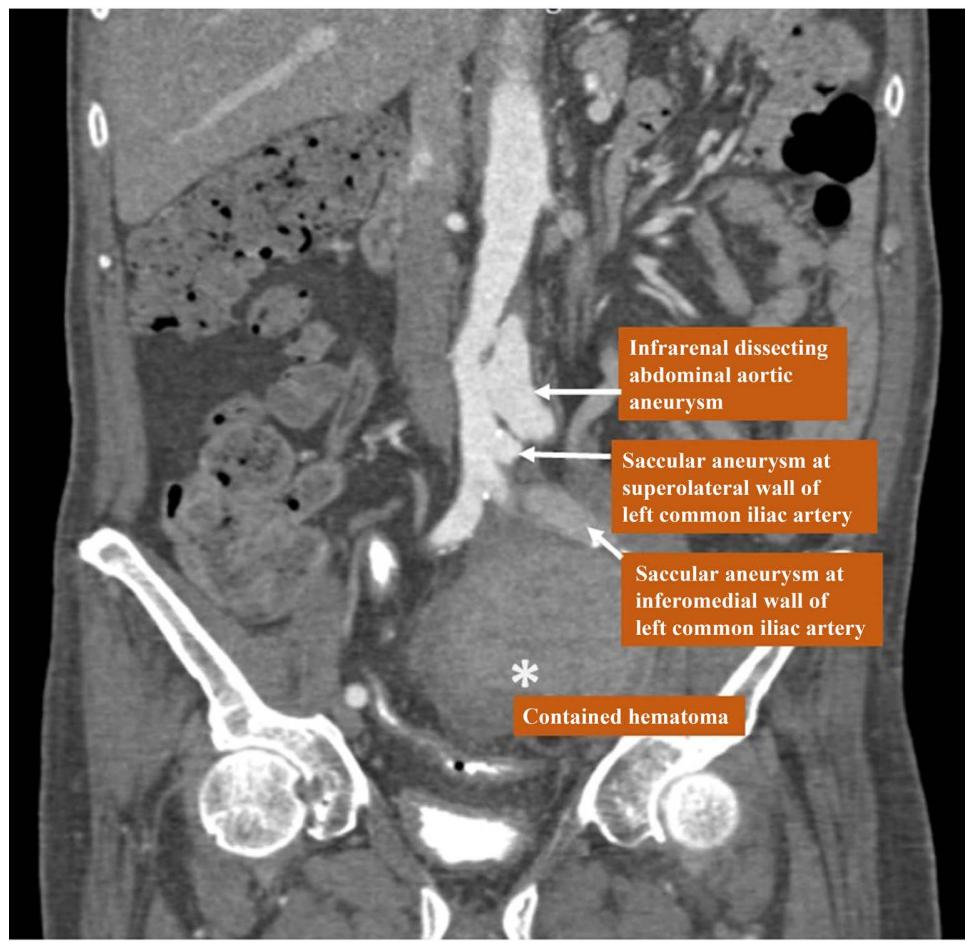

Figure 1. Computed tomography angiography (CTA) of abdomen, coronal view shows presence of two contrast filled lobulated saccular anurysms seen at left common iliac artery. The smaller aneurysm at superolateral wall of left common iliac artery measures 1.0 $\mathrm{cm} \times 0.8 \mathrm{~cm}(\mathrm{AP} \times \mathrm{WT})$ with the defect opening measures $0.8 \mathrm{~cm}$ in diameter. Another larger aneurysm arising from inferomedial wall of left common iliac artery measures 5.7 $\mathrm{cm} \times 7.7 \mathrm{~cm} \times 6.4 \mathrm{~cm}(\mathrm{AP} \times \mathrm{WT} \times \mathrm{CC})$ with the defect opening measures $1.2 \mathrm{~cm}$ in diameter. The area marked with ${ }^{\star}$ shows contained hematoma from the aneurysms. CTA abdomen also shows infrarenal dissecting abdominal aortic aneurysm (AAA) measures $3.2 \mathrm{~cm}$ in its widest diameter. The true and false lumens measure $1.6 \mathrm{~cm}$ in diameter respectively.

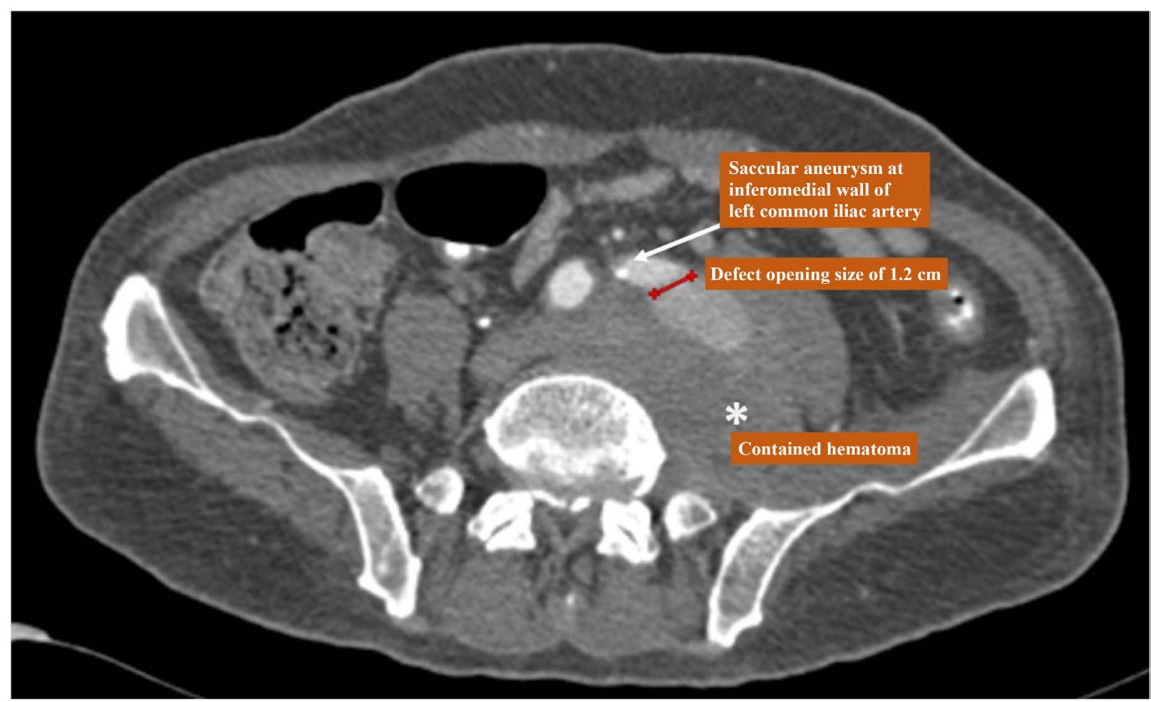

Figure 2. Computed tomography angiography (CTA) of abdomen, axial view shows presence of saccular aneurysm arising from inferomedial wall of left common iliac artery measures $5.7 \mathrm{~cm} \times 7.7 \mathrm{~cm} \times 6.4 \mathrm{~cm}(\mathrm{AP} \times \mathrm{WT} \times \mathrm{CC})$ with the defect opening measures 1.2 $\mathrm{cm}$ in diameter. The area marked with ${ }^{*}$ shows contained hematoma from the aneurysm. 
based on a susceptibility test, he was discharged with an extended course of oral ciprofloxacin. However, during the third postoperative month, the patient presented with extensive left lower limb gangrene and severe septicaemia. The patient later died despite extensive medical and surgical intervention.

\section{Discussion}

Invasive Salmonella infections can present with a variety of clinical features and varying degrees of complications, particularly in high-risk patients. These patients are notably immunocompromised and warrant a comprehensive evaluation. Clinicians caring for these patients need to increase their awareness, improve their clinical acumen, and should anticipate and manage any potential complications during the course of the disease (Table 2).

This case report is unique and relevant. The patient presented with severe Salmonella bacteraemia and extensive deep vein thrombosis and later developed multiple saccular mycotic aneurysms of the left common iliac artery. A thorough evaluation did not reveal other potential causes for the extensive deep vein thrombosis, except the severe Salmonella bacteraemia. Severe infection inducing a hypercoagulable state and thrombosis may explain the development of extensive deep vein thrombosis [12]. Atypical manifestations of invasive Salmonella infection with deep vein thrombosis have been previously reported but are notably rare [9] [10] [11]. Another possible explanation could be the compression of the ipsilateral iliac vein by a mycotic aneurysm arising from the left common iliac artery; however, this was not evident on a CTA scan of the abdomen. It would have been interesting if thrombophilia panels had been used to investigate the possibility of inherited or autoimmune causes of venous thrombosis. Thrombophilia panels were not done for this patient as inherited or autoimmune prothrombotic conditions seemed unlikely based on his negative family and personal history

Table 2. Patients with enhanced risk factors to develop invasive Salmonella bacteraemia.

\begin{tabular}{cc}
\hline Category & Risk Factor \\
\hline Non-modifiable & Extreme age group (Age $>65$ years old and neonates) \\
\hline Chronic smoking & Hypertension \\
Cardiovascular & Poorly controlled diabetes mellitus \\
Previous history of stroke \\
Hyperlipidaemia \\
Congestive heart failure \\
Long term steroid usage \\
Rheumatological disease \\
Liver cirrhosis \\
End stage renal failure \\
Immunocompromised State \\
Acquired Immunodeficiency Syndrome \\
Active Malignancy \\
Recent Chemotherapy
\end{tabular}


of thromboses. The extensive venous thrombosis was strongly suggestive of severe invasive Salmonella bacteraemia, a sepsis-induced hypercoagulable state and possible external compression of the ipsilateral iliac vein from the large mycotic aneurysm, causing venous stasis and thrombosis. Although the adjunctive clinical history and inflammatory markers were strongly suggestive of the extensive venous thrombosis because of severe salmonellosis and external compression, without additional thrombophilia screening, it was difficult to arrive at a definite conclusion that this was indeed the case.

It is possible that the patient had developed a mycotic aneurysm earlier, but this was missed during the initial admission and assessment, as the focus remained largely on the extensive left lower limb deep vein thrombosis. Later, the development of enlargement of the mycotic aneurysms with contained hematoma led to severe and persistent lower back pain along with a concomitant decrease in haemoglobin level. This led to further evaluation and a final diagnosis of multiple saccular mycotic aneurysms of the left common iliac artery. It is very difficult and challenging to determine whether deep vein thrombosis or mycotic aneurysms was the first potential complication of the invasive Salmonella bacteraemia. This case highlights the importance of closely monitoring a patient's progress and re-evaluating the initial diagnosis whenever there is a clinical doubt, especially when more information becomes available or if there is a change in the patient's condition. This case report is unique because mycotic aneurysms of the iliac artery are rarely reported in the literature, with previous reported cases of mycotic aneurysms predominantly occurring in the abdominal aorta [13] [14].

Salmonella has a strong affinity to adhere to damaged vessel walls, which causes inflammation, destruction, and localised abnormal dilatation, ultimately leading to mycotic aneurysm formation [2]. The process of infective endarteritis is postulated to be enhanced in patients with increased risk for developing atherosclerosis [2] [13]. Therefore, in such patients, imaging examinations should be performed to identify endovascular complications. Although mycotic aneurysms are a rare occurrence, once they develop, they can be severe and expand rapidly, leading to rupture, contained hematoma, and abscess formation. This patient did report poorly controlled diabetes, hypertension, dyslipidaemia, and possibly undiagnosed coronary artery disease, which increased his risk of atherosclerosis. The adjunctive clinical and biochemical markers, such as persistent lower back pain, increased inflammatory markers, and decreasing haemoglobin levels, prompted further investigation and evaluation of the mycotic aneurysms using CTA of the abdomen. Extensive venous thrombosis is life-threatening, and it becomes even more challenging when venous thrombosis and mycotic aneurysms coexist. This patient had aneurysms at multiple anatomical sites with contained hematoma that made the management of the venous thrombosis difficult. An inferior vena cava filter was inserted to prevent further embolization.

Currently, medical treatment for mycotic aneurysms alone has no lasting curative effect. Owing to aneurysmal rupture, formation of septic emboli, recurrent bacteraemia, abscess formation, and multi-organ failure, mortality may reach 
$100 \%$. Therefore, treatment should involve a combination of intensive targeted antibiotic therapy and surgical interventions, such as open vascular surgery or EVAR, to prevent aneurysmal rupture and remove the infected vessel foci. As the patient was unfit for open vascular surgery, he was offered EVAR. The EVAR is beneficial in preventing lethal aneurysmal rupture in the acute phase but provides less protection from recurrent sepsis complications [15] [16]. Despite achieving negative cultures from the peripheral blood before and after EVAR and continued intensive antibiotic therapy, the patient experienced septic complications and succumbed to these complications. Endovascular cultures may have provided more accurate results than peripheral blood cultures. Clinicians must remember that peripheral blood cultures have poor sensitivity and specificity for mycotic aneurysms, particularly when the patient has received long-term antibiotic therapy. In such patients, negative cultures do not guarantee elimination of the foci of infection. However, this patient was already at an increased risk for mortality given his older age, poorly controlled comorbidities, and the delay in seeking care at a facility during the initial illness.

\section{Conclusion}

The main observations from this case report are first, clinicians should be vigilant and consider potential endovascular complications arising from Salmonella bacteraemia in patients with multiple comorbidities. Second, Salmonella bacteraemia can present with rare, atypical manifestations such as deep vein thrombosis, either in isolation or with infective endarteritis. Clinicians need to be aware of this association, and such cases need to be reported to the scientific community. With more reported cases, identification of the temporal relationship between Salmonella bacteremia, infective endarteritis and deep vein thrombosis will be eased in the future. Third, among high-risk patients, Salmonella-induced mycotic aneurysms are associated with exaggerated morbidity and mortality. These patients may have severe medical conditions that reduce the benefits of open vascular surgery. Currently, treatment options available for such patients are limited and prolonged medical therapy alone or in combination with EVAR is yet to achieve satisfactory results. Further, more in-depth research on effective treatment of severe endovascular infection among high-risk patients, where open vascular surgery is considered unsuitable, is urgently needed.

\section{Acknowledgements}

The authors would like to acknowledge the patient's daughter for generously allowing the use of clinical details and radiological imaging in this case report. We thank our colleague, Dr. Suhaila Bt Bohan from radiology department for her expertise and assistance in selection of the suitable radiological imaging.

\section{Data Availability}

The patient data used to support the findings of this case report are available 
from the corresponding author upon request.

\section{Informed Consent}

Written informed consent was obtained from the patient's daughter for the publication of this article (including a case history and radiographic imaging).

\section{Author's Contribution}

MS was directly involved in the treatment of the patient, literature search, and scientific writing. APR participated in providing expert opinion on patient care and review of the manuscript.

\section{Conflicts of Interest}

The authors declare that there is no conflict of interest regarding the publication of this article.

\section{References}

[1] Fernández Guerrero, M.L., Aguado, J.M., Arribas, A., Lumbreras, C. and de Gorgolas, M. (2004) The Spectrum of Cardiovascular Infections Due to Salmonella enterica: A Review of Clinical Features and Factors Determining Outcome. Medicine, 83, 123-138. https://doi.org/10.1097/01.md.0000125652.75260.cf

[2] Guo, Y., Bai, Y., Yang, C., Wang, P. and Gu, L. (2018) Mycotic Aneurysm Due to Salmonella Species: Clinical Experiences and Review of the Literature. Brazilian Journal of Medical and Biological Research, 51, e6864. https://doi.org/10.1590/1414-431x20186864

[3] Hung, Y.M. (2005) Salmonella enterica cholerasuis Bacteremia and Mycotic Aneurysm of Abdominal Aorta in a Hemodialysis Patient. Intensive Care Medicine, 31, 1594. https://doi.org/10.1007/s00134-005-2749-3

[4] Fernández Guerrero, M.L., Torres Perea, R., Gómez Rodrigo, J., Núñez García, A., Jusdado, J.J. and Ramos Rincón, J.M. (1996) Infectious Endocarditis Due to NonTyphi Salmonella in Patients Infected with Human Immunodeficiency Virus: Report of Two Cases and Review. Clinical Infectious Diseases, 22, 853-855.

[5] Galofré, J., Moreno, A., Mensa, J., Miró, J.M., Gatell, J.M., Almela, M., et al. (1994) Analysis of Factors Influencing the Outcome and Development of Septic Metastasis or Relapse in Salmonella Bacteremia. Clinical Infectious Diseases, 18, 873-878.

[6] Hohmann, E.L. (2001) Nontyphoidal Salmonellosis. Clinical Infectious Diseases, 32, 263-269.

[7] Pirvu, A., Bouchet, C., Garibotti, F.M., Haupert, S. and Sessa, C. (2013) Mycotic Aneurysm of the Internal Carotid Artery. Annals of Vascular Surgery, 27, 826-830. https://doi.org/10.1016/j.avsg.2012.10.025

[8] Lee, C.H., Hsieh, H.C., Ko, P.J., Chou, A.H. and Yu, S.Y. (2014) Treatment of Infected Abdominal Aortic Aneurysm Caused by Salmonella. Annals of Vascular Surgery, 28, 217-226. https://doi.org/10.1016/j.avsg.2013.02.021

[9] Mohanty, S., Bakshi, S., Gupta, A.K., Kapil, A., Arya, L.S. and Das, B.K. (2003) Venous Thrombosis Associated with Salmonella: Report of a Case and Review of Literature. Indian Journal of Medical Sciences, 57, 199-203.

[10] Jurić, K., Pongrac, B., Cavrić, G., Mihalić, S.N., Tesanović, S.J., Nassabain, K., et al. 
(2009) Rijetke Komplikacije Infekcije Salmonelom Enteritidis [Rare Complications of Salmonella Enteritidis Infection]. Lijec Vjesn, 131, 31-32.

[11] Carey, J., Buchstein, S. and Shah, S. (2001) Septic Deep Vein Thrombosis Due to Salmonella Johannesburg. Journal of Infection, 42, 79-80.

https://doi.org/10.1053/jinf.2000.0762

[12] Chang, R., Wu, D.K., Wei, J.C., Yip, H.T., Hung, Y.M. and Hung, C.H. (2020) The Risk of Subsequent Deep Vein Thrombosis and Pulmonary Embolism in Patients with Nontyphoidal Salmonellosis: A Nationwide Cohort Study. International Journal of Environmental Research and Public Health, 17, Article No. 3567. https://doi.org/10.3390/ijerph17103567

[13] Wong, O., Lam, T., Wong, T. and Fung, H. (2009) An Uncommon Cause of Deep Vein Thrombosis: Mycotic Aneurysm Secondary to Salmonella Arteritis. Hong Kong Journal of Emergency Medicine, 16, 255-261.

https://doi.org/10.1177/102490790901600411

[14] Soravia-Dunand, V.A., Loo, V.G. and Salit, I.E. (1999) Aortitis Due to Salmonella: Report of 10 Cases and Comprehensive Review of the Literature. Clinical Infectious Diseases, 29, 862-868.

[15] Kan, C.D., Lee, H.L. and Yang, Y.J. (2007) Outcome after Endovascular Stent Graft Treatment for Mycotic Aortic Aneurysm: A Systematic Review. Journal of Vascular Surgery, 46, 906-912. https://doi.org/10.1016/j.jvs.2007.07.025

[16] Forbes, T.L. and Harding, G.E. (2006) Endovascular Repair of Salmonella-Infected Abdominal Aortic Aneurysms: A Word of Caution. Journal of Vascular Surgery, 44, 198-200. https://doi.org/10.1016/j.jvs.2006.03.002 